the writer would suggest a simple explanation of the great difference in the results of the remaining observers. It is accepted that the cells which cause the diffraction phenonema belong to the corneal epithelium (and possibly to the endothelium). As these cells enlarge under the progress of oedema, the diameters of the halos they cause will correspondingly diminish; thus the size of the halo observed will probably differ according to the phase of the pathological process.

The writer ventures to place this note on record in the hope that others, who have the opportunity of making similar measurements on glaucomatous subjects, will do so, and will either publish their results, or be kind enough to communicate them to him. The distance at which the measurement is made is not of much importance, provided that it is sufficiently great to admit of the easy measurement of the radius of each circle. Knowing the distance from the eye to the observed source of light $(a)$ and the measurement of the halo from edge to edge (b), a simple formula enables us to calculate the angle $(\theta)$ required, viz., $\tan . \theta=\frac{a}{b}$; a reference to a logarithm table completes the calculation, and gives us the measurement of the angle in degrees. To put the matter approximately for those who desire to avoid all mathematical calculations. If the light is $10 \mathrm{ft}$. from the observing eye, the diameters of the halos will be as follows:-For $2^{\circ}, 4 \frac{1}{3}$ ins.; for $3^{\circ}, 6 \frac{1}{4}$ ins.; for $4^{\circ}, 8 \frac{1}{2}$ ins. ; for $5^{\circ}, 10 \frac{1}{2}$ ins. ; for $6^{\circ}, 12 \frac{1}{2}$ ins.; for $7^{\circ}, 14 \frac{3}{4}$ ins.; for $8^{\circ}, 17$ ins. ; for $9^{\circ}, 19$ ins.

\title{
SAFETY IN OPHTHALMIC OPERATIONS
}

BY

W. H. Simpson, M.B., Ch.B. (N.Z.), F.R.C.S.Ed.

LATE SENIOR HOUSE SURGEON, ROYAL LONDON OPHTHALMIC HOSPITAL (MOORFIELDS).

WHILE the elimination of suppuration in ophthalmic operations is probably an unattainable ideal, it is certain that the irreducible minimum has not yet been reached, and to that end our most earnest eftorts must be directed. No surgeon is perfect in his technique, and it appears reasonable that if one could practise the best points of many surgeons, one would be as nearly perfect as human error would allow. There is nothing original in the points set out below - they are a summary of lessons learned from observing the work of several British ophthalmic surgeons.

The remarks apply, of course, largely to the operation for extraction of cataract.

Preliminary examination, culture and treatment.-This is of first 
importance. The condition of the adnexa must be minutely observed, and appropriate treatment used for conjunctivitis and blepharitis till the condition is cured. Before operating, the structures must be clinically and culturally satisfactory.

Swabs should be taken from both fornices and 48-hour cultures examined by a bacteriologist. Before swabbing, it is necessary to press on the lacrymal sac in such a way as to cause the smallest quantity of fluid present in the sac to flow back through the canaliculi. Cases of mild infection limited to the sac will thus be detected, and neglect of this point may quite well explain the occurrence of suppuration in spite of a clean culture from the conjunctiva. For 24 hours previous to culture, no antiseptic treatment is applied to the eye.

A 48-hour culture is necessary for the reason that the pneumococcus cannot be excluded by a shorter incubation period and staphylococcus aureus sometimes does not exhibit the yellow of its colonies for 36-40 hours. The commonly found organisms that debar operation are: pneumococcus, pneumobacillus, streptococcus and staph. aureus. Frequently, operations are done without ill result in the presence of $\mathrm{m}$. catarrhalis and $\mathrm{b}$. Morax, but it is not good practice and they are usually quickly eradicated. A strain of staph. albus is sometimes found whose colonies show haemolysis when grown on blood agar and this should be regarded as a potentially suppurative organism. Staph. albus and b. xerosis are not generally considered to contra-indicate operation but one is justified in inquiring whether they may not be the cause of some of the cases of irido-cyclitis which not infrequently follow extraction. It is certainly not wise to operate if culture shows a profuse growth of either of these organisms.

The clearing of a conjunctival sac of harmful organisms is, in Hospital practice, usually a matter of a week's treatment, but sometimes several lotions will be tried before a clean report is obtained. Thrice daily washing out with Lot. Hydrarg. Perchlor., 1 in 10,000, and daily painting of the conjunctiva with silver nitrate solution, gr. $x$ to the ounce, will be found the most useful, but is unnecessarily drastic in cases of mild infection, which clear up well with Lot. Zinci. Sulph., gr. 1 to the ounce, three times a day, and Guttae Protargol, 10 per cent., once daily.

Obstinate infections will often clear up quickly with the use of copper sulphate drops, gr. 1 to the ounce, and an organism which has defied treatment will frequently disappear when the lacrymal sac is treated by washing out with boric lotion followed by the injection of a few drops of 5 per cent. protargol. It will save time if this treatment of the sac is carried out as a routine in all cases which have given an unsatisfactory culture.

The expression of the Meibomian glands by a blunt glass rod is, 
in some cases, a useful manoeuvre which is often overlooked and gives good results.

Of course, a certain number of cases of chronic conjunctival and lid infections never improve to such an extent as to be clinically normal and in these cases, even in the presence of a pyogenic organism, good operative results have been obtained by washing out with Lot. Hydrarg. Perchlor., 1-4000, immediately before operation. This is not sound surgery, and prolonged efforts must be made to render the eye and adnexa clinically and culturally clean before taking this risk, which is seldom justifiable in an operation of so little urgency as extraction of cataract.

The presence of a mucocele is an absolute bar to operation. The sac should be excised and the puncta sealed by touching with the cautery. This applies also to the side which is not to be the subject of operation. It is unlikely that the organisms enter the operated eye by contamination during binocular dressing, but probably the 'clean' sac is the subject of a mild infection which does not give rise to pus and would be detected if before culture the sac were firmly pressed. The frequent occurrence of a purulent conjunctivitis in the other eye at the time of a suppuration in an operated eye, is best explained by the presence at operation of a mild, undetected infection of both sacs which becomes manifest after closure of the eyes for 24 hours - the most common aftertreatment. This would appear to be an argument in favour of leaving the eyes unbandaged following operation; but if the conjunctiva, lids and sac are thoroughly investigated and found clean, no harm results from tying up the eye.

Eczematous and septic conditions of the skin of the face and any sepsis elsewhere give rise to the risk of contamination, and may generally be taken as an indication of lowered resistance to pyogenic organisms.

Beyond cutting the lashes, no preparation is necessary in a 'iclean' eye, and in order that the surgeon may form an accurate estimate of the condition of an eye, no treatment should be applied on the day of operation before he has seen the case.

All lashes should be cut close to the skin. Those not covered by the speculum are a source of risk during operation, and all provide risk during after-treatment.

The Operation.-Operator and assistants should wear sterile coats or gowns with closely fitting sleeves. Caps, completely covering the hair, avoid the risk which occurs in ophthalmic operations more than in any other, of operator's and assistants' heads coming into contact and sending a hail of organisms over the field of operation. Virulent organisms are always present in the throat and are projected many feet by speech, many yards by coughing. The wearing of masks should be an absolute rule, and 
unless the surgeon wears glasses, the mask should cover the nose as well as the mouth. The rule should apply to all in the theatre who have to do with "things sterile"--instruments, towels, porringers, etc.

A reasonably thorough cleansing of the hands and wrists with sterile liquid soap, followed by a dip for a minute in some weak antiseptic solution combined with spirit is a satisfactory hand toilet. The hands must be thoroughly dried on a sterile towel.

It is almost never safe to trust to the sterility of the patient's head towel, which is so easily contaminated during the process of putting it on-never wipe instruments on the head towel.

Swabs for use during operation should be in a sterile porringer.

The chest towel is always liable to be soiled by the patient coughing, and should not be regarded as sterile. It would appear advisable to place gauze over the lower part of the face of a bearded patient, but this simply serves, if he coughs, to guide the stream of organisms upwards towards the field of operation.

The skin of the lids and surroundings should be painted with Tr. Iodi, and skin so treated should not be regarded as sterile but as being less septic than before.

A good and well tried wash-out is Lot. Hydrarg. Perchlor., $1-10,000$. During the wash-out, one must be careful to avoid lotion overflowing on to the skin and subsequently running back into the conjunctival sac. The head should be tilted a little so that the lotion leaves the eye by the outer canthus, and the lids should be mopped dry on completing the wash-out.

Instruments, whether sterilized by boiling or by immersion in an antiseptic, must be used dry. Instruments at Moorfields are placed in racks in a dish with perforated bottom and a removable lid, and the whole dish is immersed in boiling distilled water to which a little sodium carbonate is added. After 5-10 minutes' boiling, the dish is lifted out and sufficient time is then allowed for the instruments to drain and to cool. When the lid is removed, the operator can handle the instruments with the knowledge that they are exactly in the condition in which they left the sterilizera matter of some doubt when instruments are handled in uncovered racks.

None but the simplest instruments drain throughly dry. All instruments should be shaken free of moisture to avoid the possible infection of the drop of water by the grasping finger, on its course down to the "working end" of the instrument and so on into the eye. For the same reason, the shaking must be done with the "working end" of the instrument held uppermost.

Gloves cannot be worn and hands cannot be rendered sterile, so that any part of an instrument that has been in contact with the skin cannot be considered sterile. In using double-ended 
instruments, it is not easy to avoid contamination of the end not in use at the time and on this ground, such instruments should.be discarded.

It is necessary to emphasize the mistake of touching anything with the "working end" of an instrument except the actual tissues it is meant to manipulate. Generally it is considered that a surgeon, if he keeps within the blades of the speculum, is surgically clean. The speculum is certainly a boundary to be noted, but within that again there is a boundary equally as important and not sufficiently recognized. Nothing that has touched the conjunctiva should enter the globe. If one accepts the under surface of a conjunctival flap as being generally safe, this rule can be observed in nearly all cases.

A speculum with both blades solid is the cleanest type, and can, in most cases, be used without unduly cramping the field of operation. During its insertion, the speculum is very often soiled by contact with the skin and lashes-if the latter have not been cut. The lower blade should first be inserted with the upper blade turned well away, with the lids held apart and the patient looking upwards. It is then easy to insert the upper blade in the usual manner with the patient looking downwards.

In swabbing, forceps should invariably be used for holding the swab. The smaller the swab, the less likely it is to be contaminated in use. "Very small swabs, used only once" should be the rule.

Certain manoeuvres in the performance of the cataract operation should be mentioned as hindering the entrance of organisms into the eye. A sufficiently large conjunctival flap should be aimed at -ideally, one that will seal the whole length of the section. Cutting the capsule with the knife on its way across the anterior chamber ensures that one fewer instrument enters the eye and, in skilled hands, does not increase the risks of the operation. In the expression of the lens, frequent, successive, light pressing movements on the lower half of the cornea are to be avoided, for the reason that they induce a pumping effect, causing at the wound a to and fro movement of fluid; small; no doubt, in quantity but possibly contaminated by the conjunctiva. No method of removal of soft lens matter is perfect as regards asepsis. Probably irrigation involves more risk than one or two slow strokes on the cornea, while a curette depresses slightly the posterior lip of the wound. Fluid that collects at the canthi and in the lower fornix after completion of the section should be mopped dry.

At the first dressing-24 hours after operation - the lids must be soaked in warm perchloride lotion and mopped dry before opening the eye and, during the dressing, the lids and canthi must be kept dry. Drops used must of course be sterile, and if they are sufficiently warmed, the patient will seldom "squeeze."

The observance of all these points does not mean prolonging the 
operation of cataract extraction, the safety of which must always depend largely on speed and the absence of unnecessary manipulations. One may surely hope that by the practice of a careful technique, the perçentage of suppurations will be reduced. Is it too much to hope that fewer cases will show after operation the condition of "quiet cyclitis" frequently attributed to auto-infection?

\section{ANNOTATIONS}

Diploma in Ophthalmic Medicine and Surgery of the Royal College of Physicians of London and the Royal College of Surgeons of England

There are individuals who delight in estimating the possible output of the existing coal mines, and, after calculating the time it will take for them to become exhausted, make our flesh creep with the calamities which are then likely to ensue. They overlook the fact that probably fresh coal fields will be found, and that the ingenuity of man is likely to discover new and more efficient sources of energy than that supplied by coal.

So also, at the commencement of each medical year, we meet with letters and articles in the public press giving the number of new students entering the medical profession, comparing it with the present number of practitioners, and then drawing gloomy pictures of how the profession is becoming overcrowded. These writers overlook the fact that there is a large amount of medical work being carried on by those who have not received any medical training, and that new discoveries are from time to time being made which open up entirely new fields of work for medical practitioners.

By Thomas Young's discovery of astigmatism in 1800, by Airy's introduction of cylindrical lenses for its correction in 1827, and by Donders' great work on accommodation and refraction of the eye in 1864, a new means was opened up for the relief of pain and suffering of an incalculable amount. The provision, however, of medical men competent to undertake the work it involves, even now, falls far short of that which is required. The training and experience which is sufficient to obtain a qualification for the practice of medicine does not ensure adequate skill for the correction of errors of refraction with glasses, nor for the use of the ophthalmoscope.

The Ministry of Health, soon after it was first established, recognized this and applied to the Royal College of Physicians and Surgeons in London to see what could be done to enable them, when required, to select suitable medical practitioners to carry out 